\title{
Ultrathin, Biocompatible, and Flexible Pressure Sensor with a Wide Pressure Range and Its Biomedical Application
}

\author{
Yongrok Jeong ${ }^{\mathrm{a}, \dagger}$, Jaeho Park ${ }^{\mathrm{a}}$, Jinwoo Lee ${ }^{\mathrm{b}}$, Kyuyoung Kimª, and Inkyu Park ${ }^{\mathrm{a},{ }^{*}}$ \\ Department of Mechanical Engineering ${ }^{\mathrm{a}}$ \\ Korea Advanced Institute of Science and Technology \\ 291, Daehak-ro, Yuseong-gu, Daejeon, 34141, Republic of Korea \\ RF Medical. co. Ltd. ${ }^{\mathrm{b}}$ \\ 254, Beotkkot-ro, Geumcheon-gu, Seoul, 08511, Republic of Korea
}

flexible pressure sensor, biomedical sensor, ultrathin sensor, carbon nanotube, radiofrequency ablation

\section{SUPPORTING INFORMATION}

\section{Detailed Fabrication Method}

Materials: Polyimide (PI, PI-1388, elastic modulus of $2.55 \mathrm{GPa}$ ) was purchased from VTEC (USA). Carbon nanotubes (CNT, aspect ratio: $1.3 \times 10^{3}$, bulk density: 0.03 $\left.\mathrm{g} \mathrm{mL}^{-1}\right)$ were purchased from Hyosung Corporation (South Korea). Gold (Au) and titanium (Ti) with a purity of $99.99 \%$ were purchased from Taewon Science (South Korea). Polymer for anti-fouling coating (Soilnon AF$20,1 \mathrm{wt} \%$ in $3 \mathrm{M}$ Novec 7100 Engineered Fluid) was purchased from Nicca Korea (South Korea). The pressure sensitive adhesive (PSA, MD-7) was purchased from Dow Corning (USA) and the Ethyl Acetate with a purity of $99.8 \%$, which was used to dilute the PSA, was purchased from Sigma-Aldrich (USA).

Fabrication of Electrode Film: A polyimide layer for sensor substrate (spin coating, $3000 \mathrm{rpm}, 30 \mathrm{~s}$ ) was fabricated on an 8-inch silicon wafer. The PI layer was fabricated through varnish coating and baking inside an oven. For the oven baking procedure, a solvent drying process $\left(150^{\circ} \mathrm{C}, 1 \mathrm{hr}\right)$ and imidization process $\left(260^{\circ} \mathrm{C}, 3\right.$ $\mathrm{hr}$ ) were sequentially carried out. On the substrate layer, $\mathrm{Ti} / \mathrm{Au}$ (electron beam evaporation, $30 \mathrm{~nm} / 200 \mathrm{~nm}$ ) RTD electrode was patterned using a lift-off process. After the deposition of the RTD electrode layer, the insulation layer between the RTD electrode and the pressure sensor electrode was formed with a PI (spin coating, $4000 \mathrm{rpm}$, $30 \mathrm{~s}$ ). To allow for opened contact areas, the contact region was masked with a commercial PI tape before the spin coating process. On the insulating layer, the electrodes for pressure sensor was patterned with the same condition and procedure as with the RTD electrodes. On the deposited pressure sensor electrode layer, PI protective layer with the same coating condition of PI insulation layer was coated. In order to increase the sensitivity, the electrodes were fabricated with an interdigitated electrode configuration (Figure 2(a)).

Fabrication of PI/CNT Film: By employing a 6-inch silicon wafer with a thermally grown silicon oxide layer of $1 \mu \mathrm{m}$ thickness, a silicon mold was fabricated first. Following a standard photolithography process (AZ5214E, reversal bake procedures), the mask for oxide etching was patterned. Using the formed PR pattern as the masking layer, the top oxide layer was patterned via wet etching with a buffered oxide etchant (with a volume concentration of 1:6 in DI water). This layer was used as an etch mask for silicon during TMAH anisotropic etching (TMAH purity of $25 \mathrm{wt} \%, 85^{\circ} \mathrm{C}, 10 \mathrm{~min}$ ). As a result, an inverse-micropyramidal pattern with a length of about $10 \mu \mathrm{m}$ and a height of about $7 \mu \mathrm{m}$ was formed. On the fabricated mold wafer, anti-fouling coating layer was coated by spin coating at $4000 \mathrm{rpm}$ for $30 \mathrm{~s}$, CNT was deposited (spray coating on $150{ }^{\circ} \mathrm{C}$ hot plate, 0.05 wt $\%$ CNT in IPA solution dispersed with a tip sonicator, $90 \mathrm{~mL}$ ), PI varnish was coated by spin coating at 3000 rpm for $30 \mathrm{~s}$, and CNT was soaked by PI varnish through vacuum treatment (Figure 2(b)). 
Sensor Assembly: For the sensor assembly process, a PI tape film was fabricated first. It was fabricated by coating a PI film on a 6-inch silicon wafer (spin coating, $3000 \mathrm{rpm}, 30 \mathrm{~s}$ ) and applying PSA solution on it (hand bar coating, target thickness of $10 \mu \mathrm{m}$, diluted MD-7 by ethyl acetate in a volume ratio of 1:1). The fabricated PI/CNT film was cut and transferred using the fabricated PI tape film on the electrode film (Figure 2(c)).

Fabrication of Pressure Sensor Integrated RFA Needle: After removing the PET insulating tube from commercial RFA needle (Big tip, RF medical, South Korea), the PI/CNT based pressure sensor was attached on the RFA needle by applying a diluted PSA solution, which was used in the sensor assembly process. An LPF PCB board was put into the needle handle and connected with the sensor (Figure 6(a-ii)). After the attachment of the sensor, a heat shrinkable PET tube (Nordson Medical, USA) was applied on the needle again for the electrical insulation and the protection of the sensor from the environment (Figure 2(d)).

\section{Detailed Experimental Method}

Pressure Sensor Characterization: The sensor characterization was performed by the setup shown in Figure S4(a). A SM-100 load cell (Interface, Inc., USA) was used as the reference force sensor. The static pressure response test was performed in the range of 10$500 \mathrm{kPa}$. A linear actuator (Physik Instrumente GmBH \& Co. KG, Germany) was set to move with a speed of 0.7 $\mu \mathrm{m} \mathrm{s}^{-1}$ for the static pressure response test and the temperature compensation test. The linear actuator was set to move with a speed of $50 \mu \mathrm{m} \mathrm{s}^{-1}$ for the dynamic pressure response test.

Temperature Compensation Test: The temperature compensation test was performed on the modified experimental setup used in the static pressure response test (Figures S4(a)). A ceramic heater was added between the PI/CNT based sensor and the stage (Figure S4(b)). The ceramic heater maintained a constant temperature during the experiment. 


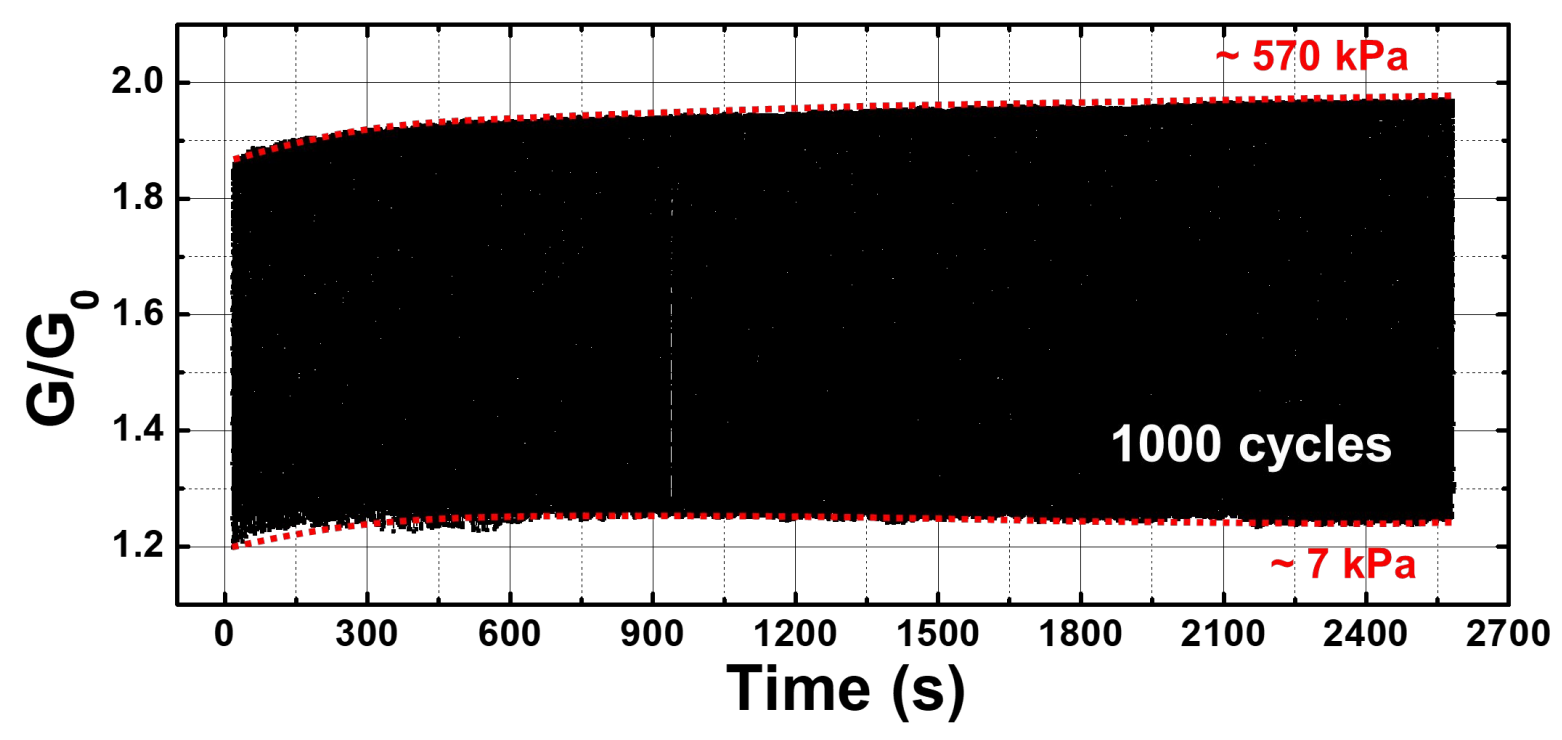

Figure S1. Stability test result of the fabricated pressure sensor. The stability test was performed within the pressure range of $7 \mathrm{kPa} \sim 570 \mathrm{kPa}$. 


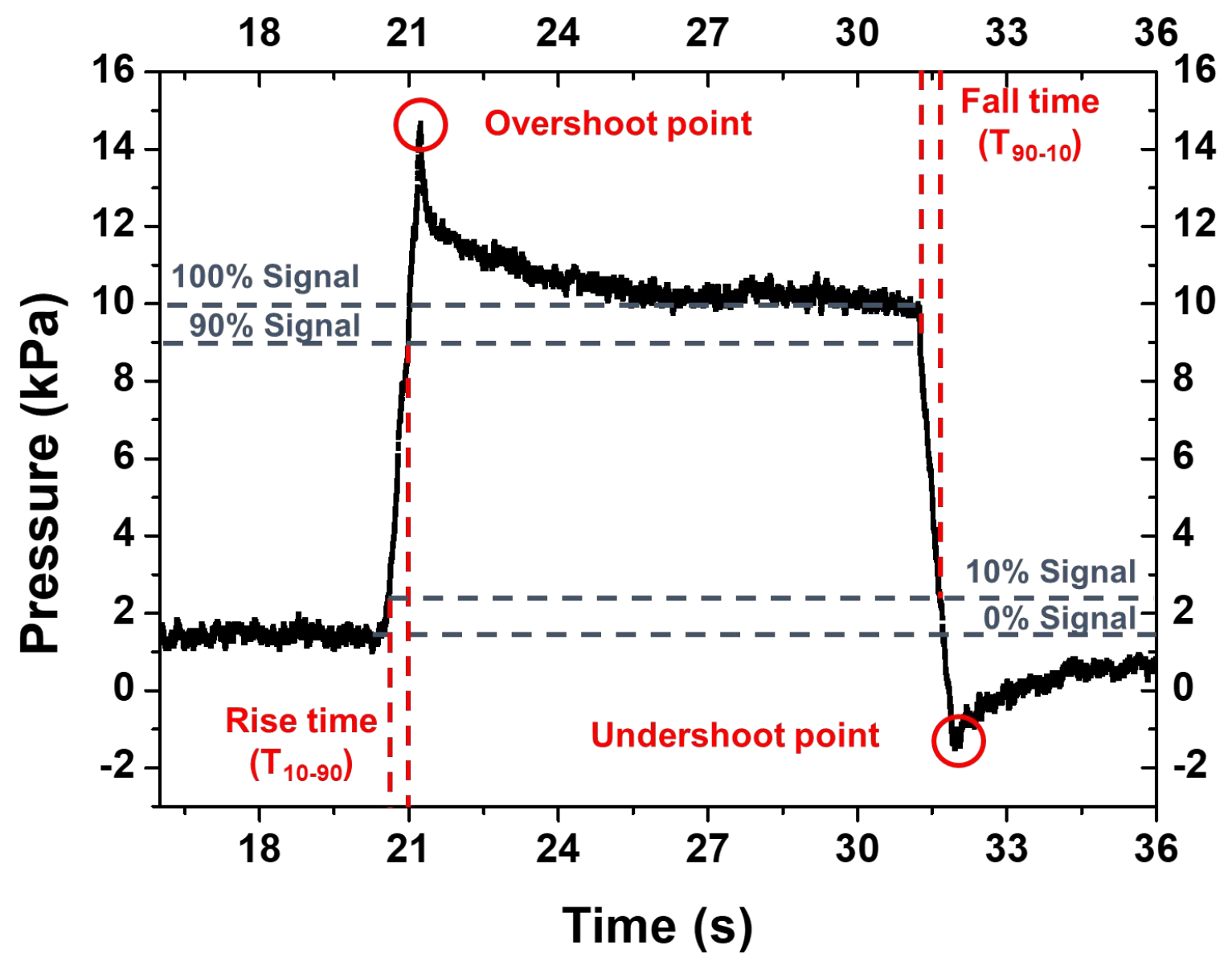

Figure S2. Definitions of the rise and fall times: Definition for the 10-90 rise time $\left(T_{10-90}\right)$ and 90-10 fall time $\left(T_{90-10}\right)$ are used for the calculation. $T_{10-90}$ was defined as the time difference between the moment with a signal of $10 \%$ and the moment with a signal of $90 \%$. Similarly, $T_{90-10}$ was defined as the time difference between the moment with a signal of $90 \%$ and the moment with a signal of $10 \%$. 


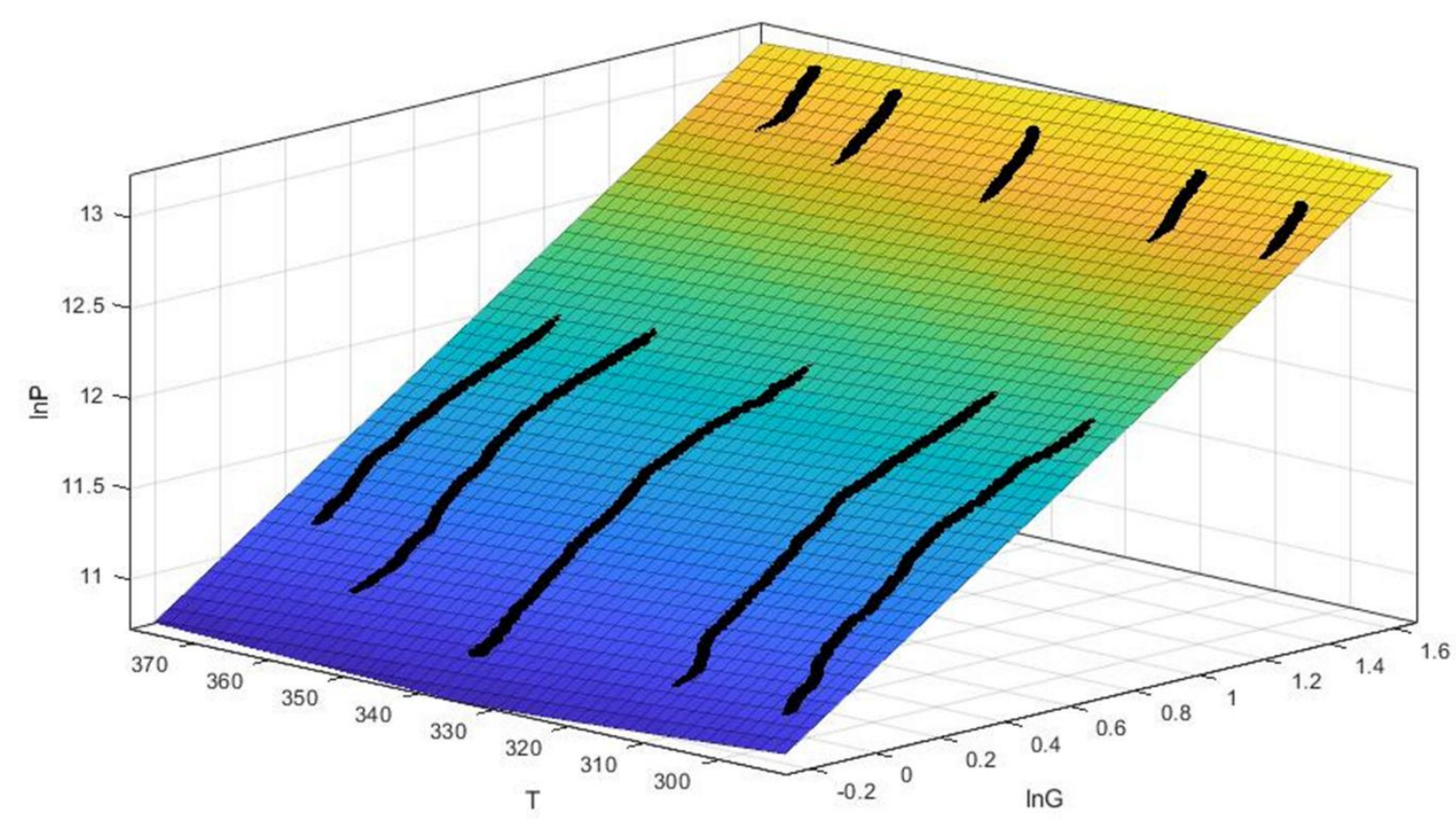

\section{$\log P=a+b \log G+c T^{-1}$}

Figure S3. Calibration result for the temperature compensation experiment. With five different temperature condition, loading curve of the sensor was measured and analyzed. 

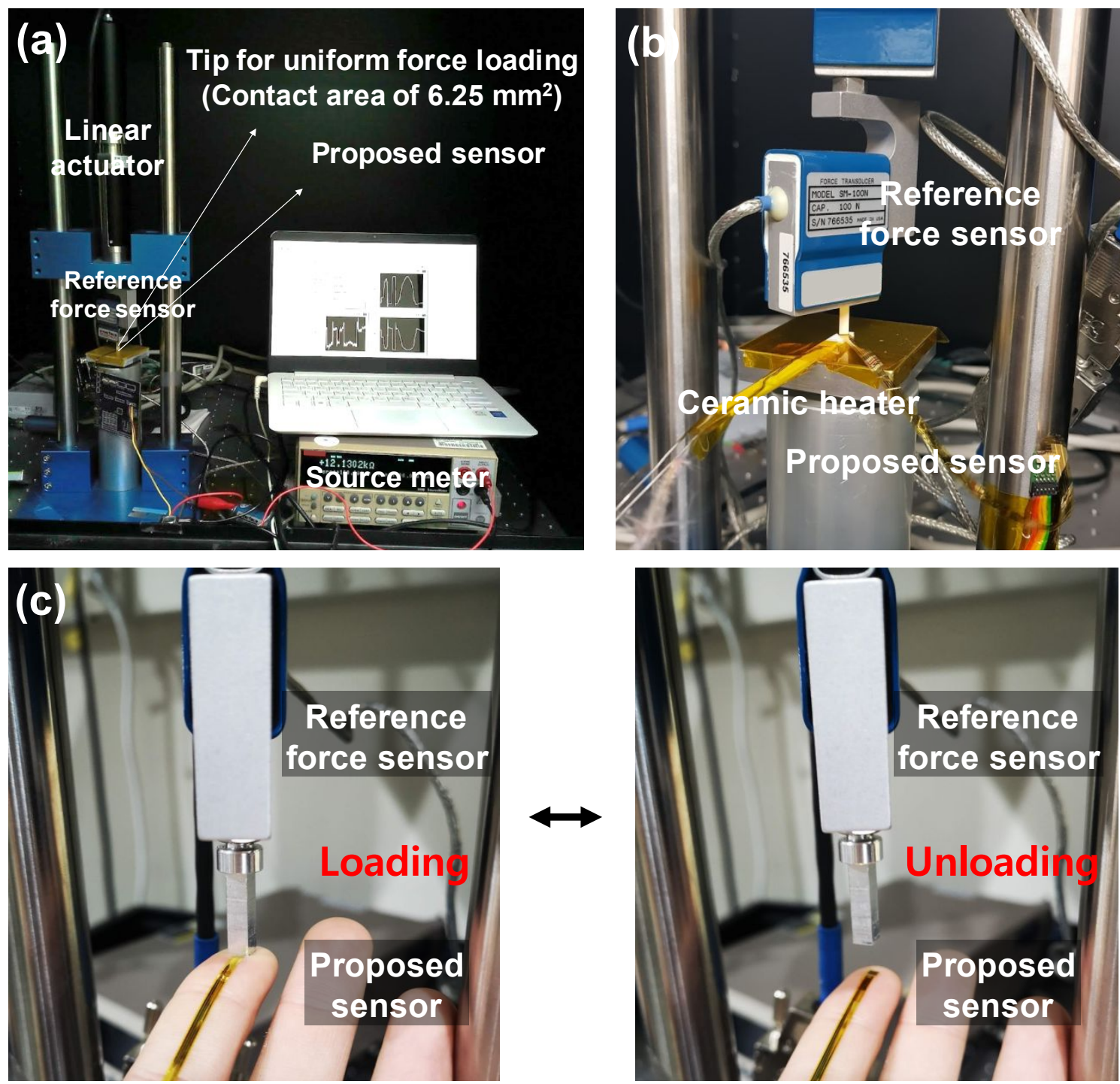

Figure S4. Experimental setup for the characterization of the PI/CNT based sensor: (a) Experimental setup for the static pressure response test; (b) Modified experimental setup in which a ceramic heater was added in between the sensor and the stage for temperature compensation test; (c) Experimental setup for the fingertip pressure measurement. 


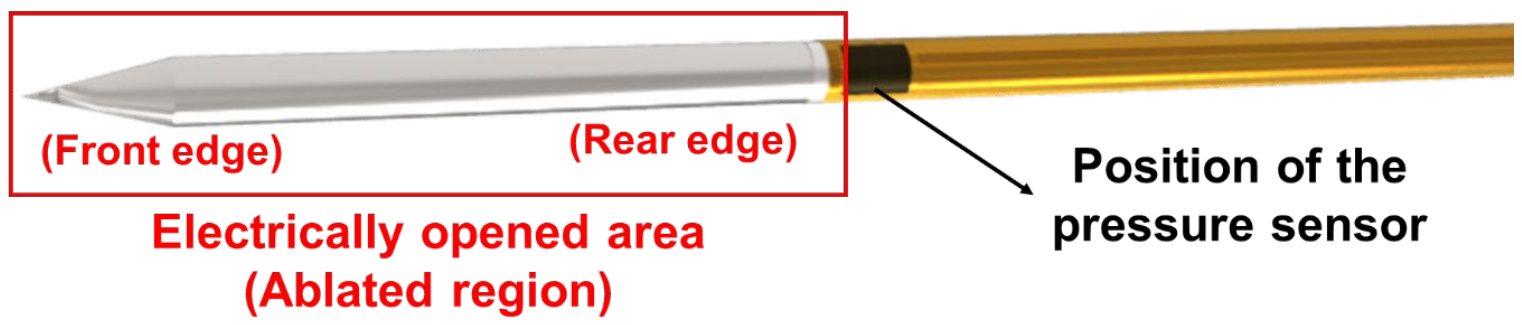

Figure S5. Detailed view for the pressure sensor integrated RFA needle 


\section{Caption for Supporting Movie}

Movie S1. Supporting movie for the occurrence of the steam popping and its detection through the proposed pressure sensor. 